УДК 517.9

\title{
On the Fredholm property for the steady Navier-Stokes equations in weighted Hölder spaces
}

\author{
Andrei A. Parfenov, \\ Alexander A.Shlapunov* \\ Institute of Mathematics and Computer Science, \\ Siberian Federal University, \\ Svobodny 79, Krasnoyarsk, 660041
}

Russia

Received 10.05.2018, received in revised form 10.06.2018, accepted 20.07.2018

We prove that the steady Navier-Stokes equations induce a Fredholm non-linear map on the scale of Hölder spaces weighted at the infinity.

Keywords: steady Navier-Stokes Equations, non-linear Fredholm operators, weighted Hölder spaces. DOI: 10.17516/1997-1397-2018-11-5-659-662.

The theory of nonlinear Fredholm operators by S. Smale [3] provides an approach to obtain generic results on the uniqueness and/or existence for nonlinear equations in Banach spaces. We recall that a bounded linear operator $\mathcal{L}$ in Banach spaces $\mathcal{X}$ and $\mathcal{Y}$ is called Fredholm if its kernel and cokernel are finite-dimensional and its range is closed. Then a nonlinear operator $\mathcal{N}$ is Fredholm if at every point $x \in \mathcal{X}$ its derivative (i.e. the principal linear part) $\mathcal{N}_{x}^{\prime}$ possesses the Fredholm property. The most advanced results were obtained for the so called proper operators (a map is proper if the inverse image of a compact set is compact). For instance, using results on proper Fredholm maps from [3], J.C. Saut and R. Temam proved the generic uniqueness theorem for the steady version of the Navier-Stokes equations on the scale of the Sobolev spaces, see [4]. Recently, A. Shlapunov and N. Tarkhanov [2] proved that the evolution Navier-Stokes equations induce a Fredholm open injective map on the scale of the Hölder spaces over the strip $\mathbb{R}^{n} \times[0, T]$, $T>0, n \geqslant 2$, weighted at the infinity with respect to the space variables. In the present short note we prove that the steady Navier-Stokes type equations induce a Fredholm map on the scale of the Hölder spaces over $\mathbb{R}^{n}, n \geqslant 3$, weighted at the infinity.

Namely, let $\mathbb{Z}_{\geqslant 0}$ be the set of all natural numbers including zero, and let $\mathbb{R}^{n}$ be the Euclidean space of dimension $n \geqslant 3$ with coordinates $x=\left(x^{1}, \ldots, x^{n}\right)$. Following [2], we denote by $C_{\delta}^{s, 0}$ the space of all $s$ times continuously differentiable functions on $\mathbb{R}^{n}$ with finite norm

$$
\|u\|_{C_{\delta}^{s, 0}}=\sum_{|\alpha| \leqslant s} \sup _{x \in \mathbb{R}^{n}}(1+|x|)^{(\delta+|\alpha|) / 2}\left|\partial^{\alpha} u(x)\right| .
$$

For $0<\lambda<1$, we introduce

$$
\langle u\rangle_{\lambda, \delta}=\sup _{\substack{x \neq y \\|x-y| \leqslant|x| / 2}}\left(\max \left(1+|x|^{2}, 1+|y|^{2}\right)\right)^{(\delta+\lambda) / 2} \frac{|u(x)-u(y)|}{|x-y|^{\lambda}} .
$$

*ashlapunov@sfu-kras.ru

(C) Siberian Federal University. All rights reserved 
We define $C_{\delta}^{s, \lambda}$ to consist of all $s$ times continuously differentiable functions on $\mathbb{R}^{n}$, such that

$$
\|u\|_{C_{\delta}^{s, \lambda}}=\|u\|_{C_{\delta}^{s, 0}}+\sum_{|\alpha| \leqslant s}\left\langle\partial^{\alpha} u\right\rangle_{\lambda, \delta+|\alpha|}+\|u\|_{C^{s, \lambda}\left(\bar{B}_{1}\right)}<\infty
$$

where $C^{s, \lambda}\left(\bar{B}_{1}\right)$ is the space of Hölder functions in the unit closed ball $\bar{B}_{1}$ centered at the origin. These are Banach spaces for all $s \in \mathbb{Z}_{\geqslant 0}$ and all $0 \leqslant \lambda<1$.

Denote by $\Lambda^{q}$ the bundle of the differential forms of degree $q$ and let $C_{\delta, \Lambda^{q}}^{s, \lambda}$ stand for the spaces of the differential forms of degree $q$ with the coefficients in $C_{\delta}^{s, \lambda}$.

Problem 1 Let $s \geqslant 2$ and $\delta>0$. Given form $f \in C_{\delta+2, \Lambda^{1}}^{s-2, \lambda}$ find a form $u \in C_{\delta, \Lambda^{1}}^{s, \lambda}$ and a function $p \in C_{\delta+1}^{s-1, \lambda}$ satisfying

$$
\left\{\begin{aligned}
-\mu \Delta u+\mathbb{D} u+d_{0} p & =f \\
d_{0}^{*} v & =0
\end{aligned}\right.
$$

where $\mu$ is a positive real number, $\Delta$ is the Laplace operator, $d_{q}$ is the de Rham differential on $q$-forms, $d_{q}^{*}$ is its formal adjoint and $\mathbb{D} u=\sum_{j=1}^{n} u_{j} \partial_{j} u$.

For $n=3$ the de Rham differentials give: $d_{0}=\nabla, d_{1}=$ rot, $d_{2}=$ div where $\nabla$ is the gradient operator, rot is the rotation operator and div is the divergence operator in $\mathbb{R}^{n}$. Hence Problem 1 is precisely the steady Navier-Stokes equations on the scale $C_{\delta}^{s, \lambda}$ if $n=3$.

Now, integration by parts yields that, for $\delta>n-2$, we have

$$
(f, 1)_{L^{2}\left(\mathbb{R}^{n}\right)}=0,
$$

for any form $f \in C_{\delta+2, \Lambda^{1}}^{s-2, \lambda}$ admitting a solution $(u, p)$ to Problem 1. Clearly, the weighted space $C_{\delta}^{s, \lambda}$ with $\delta>0$ corresponds to the one point compactification of $\mathbb{R}^{n}$ and then Problem 1 is similar to the steady Navier-Stokes equations in the periodic case (or, the same, on a torus) and (1) is similar to [4, condition (2.3)]. We will always assume that (1) is fulfilled if $\delta>n-2$.

On the next step, using Hodge theory for the de Rham complex over weighted spaces (see [2]), we reduce the Navier-Stokes equation for the velocity $u$ to the equation with respect to vorticity $d_{1} u$ (cf. [1] on the scale of Sobolev spaces). With this purpose, we note that ker $d$ will stand for solutions of the equation $d u=0$ in $\mathbb{R}^{n}$ in the sense of distributions. We denote by $R_{\delta+1, \Lambda^{q+1}}^{s-1, \lambda}(d)$ the range of the operator $d: C_{\delta, \Lambda^{q}}^{s, \lambda} \rightarrow C_{\delta+1, \Lambda^{q+1}}^{s-1, \lambda}$. According to [2, Corollary 3.11], the space $R_{\delta+1, \Lambda^{q+1}}^{s-1, \lambda}(d)$ coincides with $C_{\delta+1}^{s-1, \lambda} \cap \operatorname{ker} d_{q+1}$ if $\delta \in(0, n-1)$; it consists of elements $f \in C_{\delta+1}^{s-1, \lambda} \cap \operatorname{ker} d_{q+1}$ satisfying

$$
\left(f, d_{q} h_{j}\right)_{L^{2}\left(\mathbb{R}^{n}\right)}=0,
$$

for all harmonic homogeneous polynomials $h_{j}$ of degree $j$ in $\mathbb{R}^{n}$ with $0 \leqslant j \leqslant m+1$ if $\delta \in$ $(n-1+m, n+m), m \in \mathbb{Z}_{\geqslant 0}$.

Now, let $\wedge$ be the exterior product on the differential forms and let $\star$ be the Hodge star operator on the differential forms, induced by identity $d x_{I} \wedge \star d x_{I}=d x$ for each differential $d x_{I}=d x_{i_{1}} \wedge \cdots \wedge d x_{i_{q}}$ with $|I|=q$. Now for $q$-form $F(x)=\sum_{|I|=q} F_{I}(x) d x_{I}$ we set

$$
(\varphi F)(x)=\int_{\mathbb{R}^{n}} \sum_{|I|=q} F_{I}(y) \varphi_{n}(x-y) d y, \Phi v(x)=\int_{\mathbb{R}^{n}} F(y) \wedge\left(d_{n-q-1}\right)_{y}^{*}\left(\sum_{|I|=q} \varphi_{n}(x-y) \star d y_{I}\right),
$$

where $\varphi_{n}(x-y)$ is the standard fundamental solution of the Laplace operator in $\mathbb{R}^{n}$. The behaviour of these potentials on the weighted Hölder spaces were investigated in [2, §3]. Now, for 1 -for $g$ we set

$$
\mathbb{G} g(x)=\star(\star g \wedge \Phi g) .
$$


Problem 2 Let $s \geqslant 3$ and $\delta>0$. Given form $f \in C_{\delta+2, \Lambda^{1}}^{s-2, \lambda}$ (satisfying (1) if $\delta>n-2$ ) find a form $g \in R_{\delta+1, \Lambda^{2}}^{s-1, \lambda}$ satisfying

$$
g-(1 / \mu) \varphi \mathbb{G} g=\varphi d_{1} f
$$

Now we may achieve the main theorems of our note.

Theorem 1. Let $n \geqslant 3, s \in \mathbb{N}, s \geqslant 3$ and $1<\delta<n / 2$ with $2 \delta-n+3 \notin \mathbb{Z} \geqslant 0$. Then Problems 1 and 2 are equivalent.

Proof. Indeed, we may write the nonlinear term $\mathbb{D}$ in the Lamb form (see, for instance, [1] or [2, Lemma 1.2]:

$$
\mathbb{D} u=d_{0}|u|^{2}+\star\left(\star d_{1} u \wedge u\right) .
$$

According to [2, Corollary 3.11], the operator $\Phi$ maps $R_{\delta+1, \Lambda^{2}}^{s, \lambda}$ continuously to $C_{\delta, \Lambda^{1}}^{s, \lambda}$ for the chosen $\delta$. Then, as $u=\Phi g$ is the unique form from $C_{\delta, \Lambda^{1}}^{s, \lambda}$ satisfying $d_{1} \Phi g=g, d_{0}^{*} \Phi g=0$ in $\mathbb{R}^{n}$, see [2], we conclude that $d \mathbb{D}=\mathbb{G} d$. As $d^{2}=0$, this proves that Problem 1 is equivalent to the following equation

$$
-\mu \Delta g+\mathbb{G} g=d_{1} f
$$

on the discussed scales of spaces. Besides, the potential $\varphi$ induces bounded linear map from the range $R_{\delta+2}^{s-2, \lambda}(\Delta)$ of the bounded operator $\Delta: C_{\delta}^{s, \lambda} \rightarrow C_{\delta+2}^{s-2, \lambda}$ to $C_{\delta}^{s, \lambda}$ where $R_{\delta+2}^{s-2, \lambda}(\Delta)$ coincides with $C_{\delta+2}^{s-2, \lambda}$ if $0<\delta<n-2$; it consists of all the elements $F \in C_{\delta+2}^{s-2, \lambda}$ satisfying

$$
\left(F, h_{j}\right)_{L^{2}\left(\mathbb{R}^{n}\right)}=0,
$$

for all harmonic homogeneous polynomials $h_{j}$ of degree $j$ in $\mathbb{R}^{n}$ with $0 \leqslant j \leqslant m$ if $\delta \in(n-2+$ $m, n+m-1), m \in \mathbb{Z}_{\geqslant 0}$ (see, for instance, [2, Theorem 3.1].

Since $\delta>1$ the potential $\varphi d_{1} f$ is a convergent integral. Moreover, as $f$ satisfies (1) if $\delta>n-2$, then using integration by parts we see that $d_{1} f$ satisfies (4) with $j=0$ and $j=1$. Hence if $\delta \neq n-2, \delta \neq n-1, \delta \neq n, 1<\delta<n+1$, the form $\varphi d_{1} f$ belongs to $C_{\delta+1}^{s-1, \lambda}$. However, as $1<\delta<n / 2$ and $n \geqslant 3$ all these conditions are fulfilled.

Similarly, integration by parts yields $\mathbb{D} u$ satisfies (1) if $2 \delta+1>n-2$. On the other hand, according to $[2$, Lemma 2.9] on the multiplication of the weighted functions, we see that $\mathbb{D}$ maps $C_{\delta, \Lambda^{1}}^{s, \lambda}$ continuously to $C_{2 \delta+1, \Lambda^{1}}^{s-1, \lambda}$. Hence, as $2 \delta-n+3 \notin \mathbb{Z}_{\geqslant 0}$, the operator $\varphi d_{1} \mathbb{D}$ maps $C_{\delta, \Lambda^{1}}^{s, \lambda}$ continuously to $C_{2 \delta, \Lambda^{2}}^{s, \lambda}$ if $2 \delta<n$. Similarly, $\varphi \mathbb{G}$ maps $R_{\delta+1, \Lambda^{2}}^{s-1, \lambda}(d)$ continuously to $C_{2 \delta, \Lambda^{2}}^{s, \lambda}$ if $2 \delta<n$ (and, by the very definition, to $R_{2 \delta, \Lambda^{2}}^{s, \lambda}(d)$ ).

Finally, as $\varphi \Delta u=u$ for each $u \in C_{\delta, \Lambda^{q}}^{s, \lambda}$ (see, for instance, [1] or [2, Theorem 3.1]) and the space $C_{\delta, \Lambda^{q}}^{s, \lambda}$ is continuously embedded to the space $C_{\delta^{\prime}, \Lambda^{q}}^{s-1, \lambda}$ for any $\delta \geqslant \delta^{\prime}$ (see [2, Theorem 2.3]), applying the potential $\varphi$ to (3) we conclude that Problems 1 and 2 are equivalent, which was to be proved.

Theorem 2. Let $n \geqslant 3, s \in \mathbb{N}, s \geqslant 3$ and $1<\delta<n / 2$ with $2 \delta-n+3 \notin \mathbb{Z}_{\geqslant 0}$. Then the continuous operator

$$
I-(1 / \mu) \varphi \mathbb{G}: R_{\delta+1}^{s-1, \lambda}(d) \rightarrow R_{\delta+1}^{s-1, \lambda}(d)
$$

is Fredholm one and the operator

$$
(1 / \mu) \varphi \mathbb{G}: R_{\delta+1}^{s-1, \lambda}(d) \rightarrow R_{\delta+1}^{s-1, \lambda}(d)
$$

is continuous and compact.

Proof. We already proved in Theorem that $\varphi \mathbb{G}$ maps $R_{\delta+1, \Lambda^{2}}^{s-1, \lambda}(d)$ continuously to $R_{2 \delta, \Lambda^{2}}^{s, \lambda}(d)$ if $2 \delta<n$. On the other hand, as the embedding $C_{\delta, \Lambda^{q}}^{s, \lambda}$ to the space $C_{\delta^{\prime}, \Lambda^{q}}^{s-1, \lambda}$ is compact for any 
$\delta>\delta^{\prime}$ we see that $\varphi \mathbb{G}$ maps $R_{\delta+1, \Lambda^{2}}^{s-1, \lambda}(d)$ compactly to $R_{\delta+1, \Lambda^{2}}^{s, \lambda}(d)$ if $2<2 \delta<n$, i.e. the second statement of the theorem is true.

Finally, as

$$
\mathbb{G}_{\mid g=g_{0}}^{\prime} F=d \star\left(\star g_{0} \wedge \Phi F\right)+d \star\left(\star F \wedge \Phi g_{0}\right)
$$

for each $F, g_{0} \in R_{\delta+1, \Lambda^{2}}^{s-1, \lambda}(d)$, we may argue as before to conclude that

$$
(\varphi \mathbb{G})_{\mid g=g_{0}}^{\prime}: R_{\delta+1, \Lambda^{2}}^{s-1, \lambda}(d) \rightarrow R_{\delta+1, \Lambda^{2}}^{s-1, \lambda}(d)
$$

is a compact linear operator. Thus the operator $I-(1 / \mu) \varphi \mathbb{G}_{\mid g=g_{0}}^{\prime}$ is Fredholm for each $g_{0} \in$ $R_{\delta+1, \Lambda^{2}}^{s-1, \lambda}(d)$ because of the famous Fredholm theorems.

The work was supported by the grant of the Ministry of Education and Science of the Russian Federation N 1.2604.2017/PCh.

\section{References}

[1] A.Bertozzi, A.Majda, Vorticity and Incompressible Flows, Cambridge University Press, Cambridge, 2002.

[2] A.Shlapunov, N.Tarkhanov, An Open Mapping Theorem for the Navier-Stokes Equations, Advances and Applications in Fluid Mechanics, 21(2018), no. 2 127-246.

[3] S.Smale, An infnite dimensional version of Sard's theorem, Amer. J. Math., 87(1965), no. 4, 861-866.

[4] R.Temam, Navier-Stokes Equations and Nonlinear Functional Analysis, 2nd ed., SIAM, Philadelphia, 1995.

\section{О свойстве Фредгольма для стационарных уравнений Навье-Стокса в весовых пространствах Гельдера}

Андрей А. Парфенов, Александр А. Шлапунов

$\overline{\text { Мъ доказываем, что стационарные уравнения Навъе-Стокса индуцирует нелинейный оператор }}$ фредгольмовского типа в весовых пространствах Гельдера.

Ключевые слова: стационарные уравнения Навъе-Стокса, нелинейные фредгольмовы операторы, весовые пространства Гелъдера. 